\title{
Tumor control and QoL outcomes of very young children with atypical teratoid/rhabdoid Tumor treated with focal only chemo-radiation therapy using pencil beam scanning proton therapy
}

\author{
Damien C. Weber - Carmen Ares - Robert Malyapa - Francesca Albertini • \\ Gabriele Calaminus · Ulrike Kliebsch • Lorentzos Mikroutsikos • \\ Petra Morach · Alessandra Bolsi · Tony Lomax $\cdot$ Ralf Schneider
}

Received: 23 June 2014 / Accepted: 26 October 2014/Published online: 2 November 2014

(C) Springer Science+Business Media New York 2014

\begin{abstract}
The aim of this analysis was to assess the early clinical results of pencil beam scanning proton therapy (PT) in the treatment of young children with non-metastatic atypical teratoid/rhabdoid tumor (ATRT) of the CNS. Fifteen children (male, $n=8,53 \%$ ) were treated with PT between May 2008 and January 2013. Mean age at diagnosis was $17.4 \pm 7.0$ months. The localization was infratentorial in $9(60 \%)$ patients. Gross total resection of the primary tumors was achieved in $7(47 \%)$ patients. The dose administered focally under sedation was $54 \mathrm{~Gy}$ (RBE). After a median follow-up of 33.4 months (range
\end{abstract}

This work has been submitted for oral presentation to the 2014 SIOP meeting, Toronto, Canada.

\footnotetext{
D. C. Weber $(\varangle) \cdot$ R. Malyapa · F. Albertini · U. Kliebsch ·

L. Mikroutsikos · P. Morach - A. Bolsi - T. Lomax ·

R. Schneider

Center for Proton Therapy, Paul Scherrer Institute, WPTA 144,

CH-5232 Villigen West, Switzerland

e-mail: damien.weber@psi.ch; damiencharles.weber@usz.ch

R. Malyapa

e-mail: robert.malyapa@psi.ch

F. Albertini

e-mail: francesca.albertini@psi.ch

U. Kliebsch

e-mail: ulrike.kliebsch@psi.ch

L. Mikroutsikos

e-mail: lorenzos.mikroutsikos@psi.ch

P. Morach

e-mail: petra.morach@psi.ch

A. Bolsi

e-mail: alessandra.bolsi@psi.ch

T. Lomax

e-mail: toni.lomax@psi.ch
}

9.7-69.2), $3(20 \%), 4(27 \%)$ and $2(13 \%)$ patients presented with local failure (LF), distant brain failure (DBF) and spinal failure (SF), respectively. Six patients died, all of tumor progression. The 2-year overall- and progressionfree survival was 64.6 and $66.0 \%$. Tumor location (supratentorial) and the extent of surgical resection (nongross total resection) were negative prognostic factors for both OS and PFS. PT was well tolerated. No grade $>2$ acute toxicity was observed. The estimated 2-year toxicityfree survival was $90 \%$. As assessed by the PedsQoL proxy, no decrease in QoL was observed after PT. We conclude that PBS PT is an effective treatment for young children with ATRT. After PT, with or without concomitant chemotherapy, two third of the patients survived $>2$ years. Acute toxicity was manageable. Longer follow-

\author{
R. Schneider \\ e-mail: ralf.schneider@psi.ch \\ D. C. Weber \\ University of Zürich, Zurich, Switzerland \\ C. Ares \\ Department of Radiation Oncology, Geneva University Hospital, \\ Geneva, Switzerland \\ e-mail: carmen.ares@hcuge.ch \\ G. Calaminus \\ Department of Pediatric Hematology and Oncology, University, \\ Hospital Münster, Münster, Germany \\ e-mail: gabriele.calaminus@ukmuenster.de \\ T. Lomax \\ Department of Physics, Swiss Institute of Technology (ETH), \\ Zurich, Switzerland
}


up and larger numbers of patients are needed to assess long-term outcomes and treatment-induced toxicity.

Keywords Atypical teratoid/rhabdoid tumor - Pencil beam scanning proton therapy · ATRT children - Brain tumor - Quality of life

\section{Introduction}

Atypical teratoid/rhabdoid tumor (ATRT) of the CNS is a rare, highly malignant and extremely aggressive embryonal neoplasm of early childhood. This tumor accounts for $1-2 \%$ of CNS pediatric tumors but up to $20 \%$ of malignant CNS neoplasms in patients younger than 3 years of age [1-3]. Administered treatments are not ATRT specific and are highly variable but typically includes multimodality treatment, namely surgery, chemotherapy and radiation therapy (RT). After this multimodality strategy, most patients suffer swift disease recurrence and death owing to tumor-progression. The mean reported survival time of these young ATRT children ranges from 6 to +18 months [4]. It is thus of paramount importance to improve tumor control and/or decrease treatment-related toxicity for these young patients.

Technical improvements in radiation therapy may improve the therapeutic ratio for these challenging patients. Unlike conventional radiotherapy, proton therapy (PT) allows for optimal dose distributions, with the added benefit of no exit dose. This absence of exit dose has triggered the rational of using protons for children with various cancer types. In a simulation study, the risk of adverse effect in pediatric patients with medulloblastoma was estimated to be the lowest with PT, when compared to photon, with or without intensity modulation, plans [5].

The physical, emotional and social aspects of the child's well-being is of prime importance for these patients, as cancer and its associated treatments are stressful and reduce the Quality of life (QoL) in children. As such, there is a need for health professionals to fully assess the treatment-impact of the multi-modality therapy administered to these young children.

We assessed the clinical results, not limited but including the recurrence pattern, toxicity and QoL, of pencil beam scanning (PBS) PT in the treatment of non-metastatic ATRT patients treated at the Paul Scherrer Institute (PSI).

\section{Methods and materials}

Patients

Between May 2008 and January 2013, 15 consecutive children with non-metastatic ATRT aged from 4.6 to 27.4 (median 18.9) months were treated with PBS PT at PSI. Eighty seven percent of these patients were $<24$ months old and $20 \%<12$ months of age. There were seven girls and eight boys. The majority $(n=12 ; 80 \%)$ of tumors were $<5 \mathrm{~cm}$. Original immunohistochemistry investigations varied in scope (Table 1). All tested tumors presented with nuclear loss of INI-1 (Table 1). In nine children, ATRTs were located in the posterior fossa. Gross total resection was defined as gross macroscopic removal of the visible tumor, as defined by the surgeon's operative notes and the absence of tumor on the postoperative imaging studies. Subtotal resection and macroscopic complete resection was performed in seven patients each. All patients underwent postoperative MRI. Second look surgery after chemotherapy, decided by a multi-disciplinary team assessing the tumor response, was performed in $3(20 \%)$ patients and another of these patients became tumor-free. Eight (53\%) patients had thus no residual disease before PT.

All patients received varied forms of chemotherapy (See Table 2 for details). Legal representatives gave consent for patients.

\section{PT planning and delivery}

All patients were immobilized using a combination of body cast and a vacuum-assisted bite-block system (Supratentorial ATRT) or thermoplastic mask (Infratentorial ATRT). Patient positioning was checked before every fraction, as published previously [6]. The GTV was defined as the macroscopic tumor identified on the brain magnetic resonance imaging (MRI) performed before the initiation of chemotherapy and PT, the residual tumor, if any, identified on the pre-PT brain MRI scan and the tumor bed identified on the planning computed tomography (CT) scan during simulation. The clinical target volume (CTV) included the GTV plus a $10 \mathrm{~mm}$ margin extension modified anatomically for microscopic involvement. The planning target volume (PTV) encompassed the CTV plus a 5-mm margin. The median planning target volume was $117.3 \mathrm{cc}$ (range 35.0-202.0).

The relative biologic effectiveness (RBE) factor for protons of 1.1 (relative to that of ${ }^{60} \mathrm{Co}$ ) was used, and proton doses were expressed in terms of Gy(RBE) $[\mathrm{Gy}(\mathrm{RBE})=$ proton Gy $\times 1.1]($ ref $)$. All patients received $54 \mathrm{~Gy}(\mathrm{RBE})$ in 30 fractions of $1.8 \mathrm{~Gy}(\mathrm{RBE})$. Patients were treated using the spot-scanning technique at the scanning gantry by using the $250-\mathrm{MeV}$ medical dedicated cyclotron. Proton dose was computed using a 3-dimensional dose calculation algorithm developed at PSI [7]. Single-field uniform dose (SFUD) plans and IM proton therapy (IMPT) plans were used sequentially at PSI.

Dose constraints to organs at risk (OARs) were determined as maximum dose (D2) of 50 and $54 \mathrm{~Gy}(\mathrm{RBE})$ to 
Table 1 Immunohistochemistry results and proliferative indexes of 15 ATRT children treated with proton therapy

\begin{tabular}{|c|c|c|c|c|c|c|c|c|c|c|c|c|}
\hline Study number & Vimentin & GFAP & EMA & Synaptophysin & Desmin & INI-1 & MAP-2 & NSE & NeuN & S100 & p53 & MIB1 $(\%)$ \\
\hline 1 & NP & NP & + & - & NP & Loss & NP & NP & NP & - & NP & NP \\
\hline 2 & ++ & NP & + & - & - & Loss & + & NP & NP & - & NP & 20 \\
\hline 3 & ++ & + & NP & + & NP & Loss & NP & NP & NP & NP & + & 80 \\
\hline 4 & ++ & + & + & - & - & Loss & + & NP & - & NP & \pm & 10 \\
\hline 5 & ++ & + & - & NP & NP & Loss & + & NP & NP & + & + & NP \\
\hline 6 & ++ & + & + & + & - & Loss & + & NP & NP & + & + & 50 \\
\hline 7 & NP & + & + & - & NP & Loss & + & + & + & NP & NP & 20 \\
\hline 8 & ++ & + & NP & + & NP & Loss & NP & NP & NP & NP & + & 50 \\
\hline 9 & NP & \pm & \pm & + & NP & Loss & + & + & NP & \pm & NP & 40 \\
\hline 10 & NP & - & + & + & NP & Loss & + & - & NP & + & NP & 20 \\
\hline 11 & ++ & - & + & NP & NP & Loss & NP & + & - & NP & NP & NP \\
\hline 12 & ++ & + & + & - & - & Loss & NP & NP & NP & NP & NP & 30 \\
\hline 13 & ++ & + & NP & NP & NP & Loss & + & NP & NP & NP & NP & 10 \\
\hline 14 & NP & + & + & + & NP & Loss & NP & NP & NP & + & NP & 50 \\
\hline 15 & ++ & - & + & - & - & Loss & - & NP & + & NP & + & 10 \\
\hline
\end{tabular}

$N P$ not performed, $\pm<10 \%$ expression, + focal expression, ++ strong expression, - negative

the center and surface of the brainstem or spinal cord, $50 \mathrm{~Gy}(\mathrm{RBE})$ to the optic chiasm, $45 \mathrm{~Gy}(\mathrm{RBE})$ to the optic nerves, mean/maximum dose (D2) of 20/30 Gy(RBE) to the lacrimal glands, mean/maximum dose (D2) of 36/45 Gy(RBE) to the cochlea's and mean/maximum dose (D2) of $7 / 10 \mathrm{~Gy}(\mathrm{RBE})$ to the lens.

PT was administered 7.2 (range 2.0-14.5) and 3.8 (range 0.6-8.0) months after the diagnosis and last surgery, respectively. Focal (i.e. non-craniospinal irradiation) only PT was delivered to all patients. Treatment plans were optimized to maximize the coverage of the GTV while observing OAR dose constraints. An example of a treatment plan for an ATRT is given in Fig. 1.

PT was delivered in 38 to 43 (median 42) days, with a 2-3 series plan, using single field uniformed dose and intensity modulated PT.

Quality of life

We investigated health-related QoL in all patients treated with PT for ATRT. Instruments included a questionnaire on life situations, PedsQoL proxy (parents' questionnaire for parents with children aged $\leq 4$ years) [8]. Parents answered a multidimensional questionnaire on child's autonomy and cognitive or behavioral difficulties and on the socio-psychological impact of the illness on their own everyday life. Higher PedsQoL proxy scores suggest better patients' QoL. The sample included all patients $(n=15)$ who had been enrolled into the multinational, multicenter prospective surveillance study of children with cancer led by the University of Münster. QoL evaluations (Table 3) were made at baseline (E1) before PT and 2 months after the end of PT (E2), so as to assess the impact of PT on QoL.

Follow-up

We used the RECIST classification to assess the radiologic outcome (partial response [PR], $\geq 30 \%$ decrease in maximum diameter), stable disease (SD $<30 \%$ decrease and $<20 \%$ increase in maximum diameter), and progressive disease ( $\mathrm{PD} \geq 20 \%$ increase in maximum diameter). Complete response (CR) was defined as the complete disappearance of the ATRT. Radiologic criteria for tumor progression, locally, distant brain and spinal, were defined as tumor growth or tumor recurrence in two consecutive MRI or CT scans. Acute toxicities were defined as those adverse events that occur from the first day of treatment through day 90 after treatment. All side effects observed after 90 days from the end of PT were considered as late adverse events. These were classified according to the National Cancer Institute Common Terminology Criteria for Adverse Events, v4.0 grading system (http://evs.nci. nih.gov/ftp1/CTCAE/CTCAE_4.03_2010-06-14_Quick Reference_5x7.pdf).

Statistical analysis

Overall (OS), progression-free (PFS) and late toxicity-free (TFS) survival times were determined from the date of the diagnosis. Survival rates were calculated using the actuarial method of Kaplan-Meier. Observations were censored on 


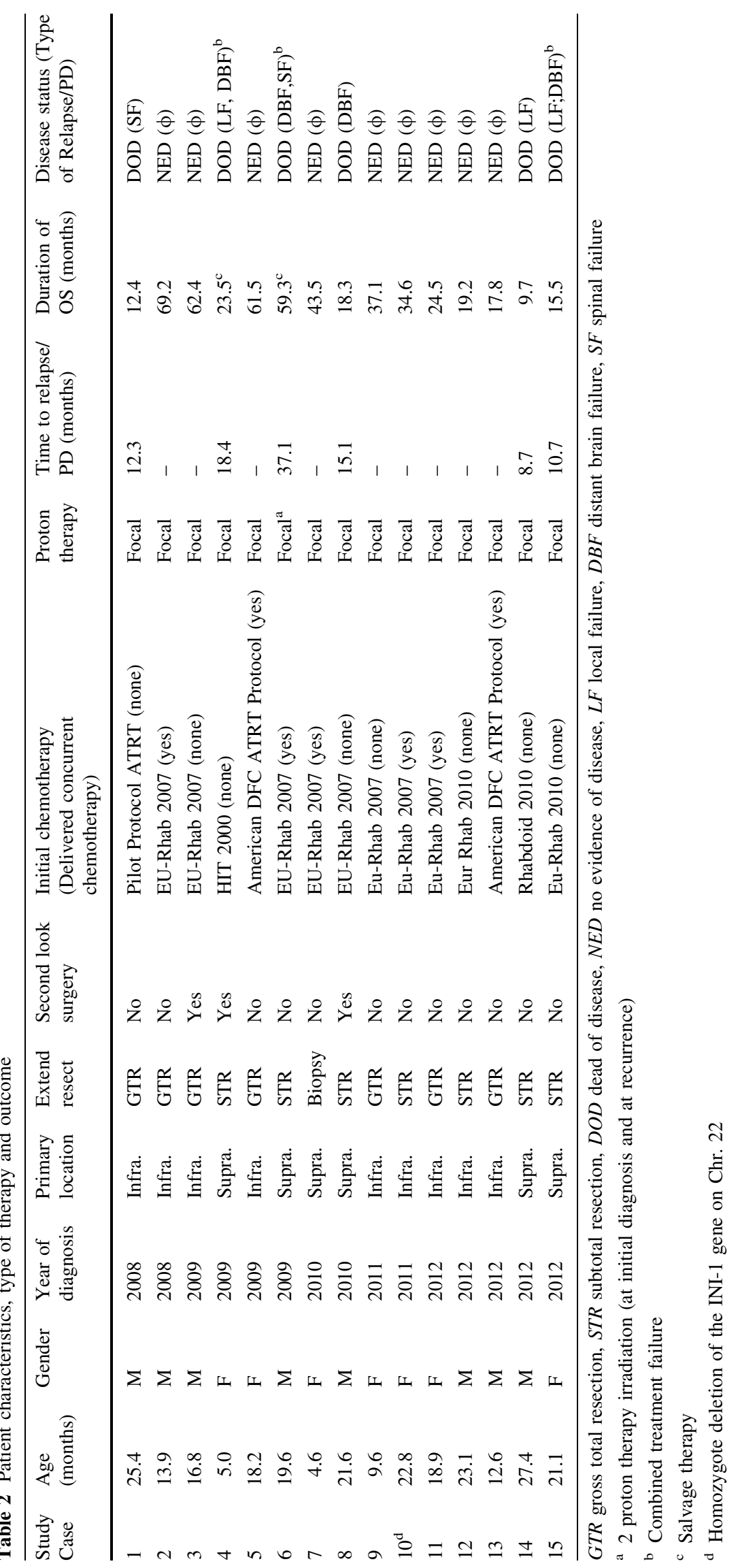




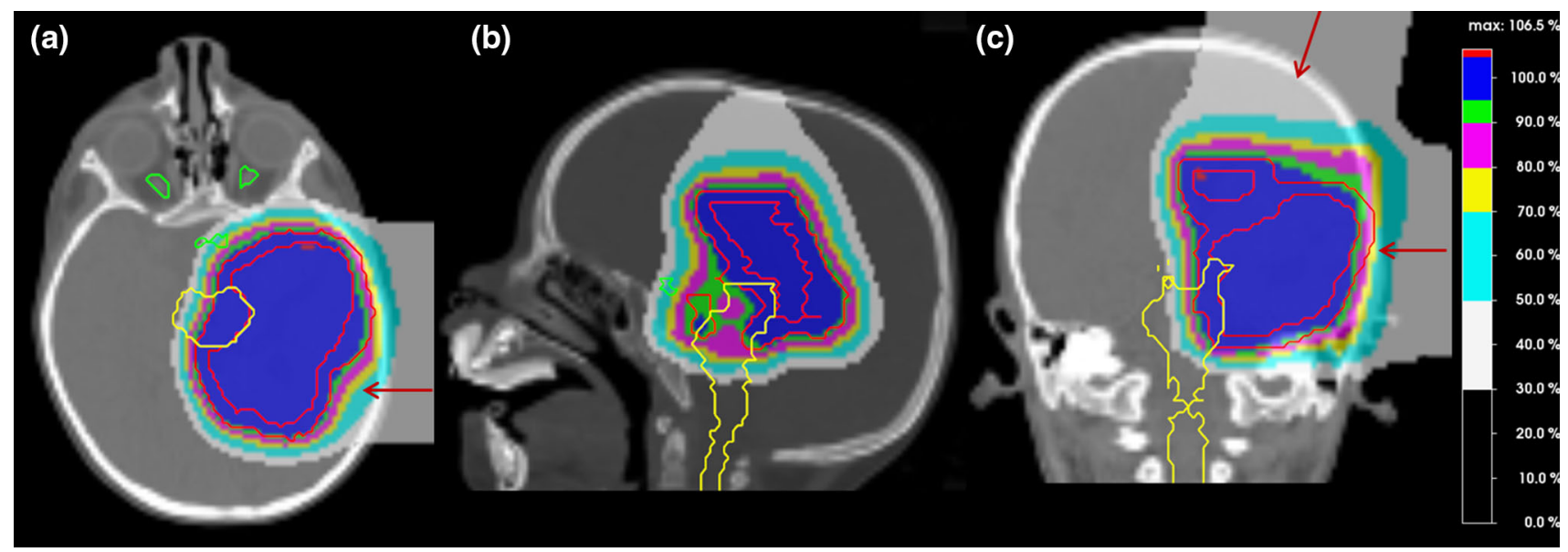

Fig. 1 Dose distribution of a treatment plan superimposed on CT images of a patient with an supratentorial ATRT, a coronal, b sagital and $\mathbf{c}$ coronal views. Note the rapid dose decline between the target and non-target volumes and the optional sparing of contro-lateral brain (coronal and axial slices). The isodose contours are represented by the color-wash (corresponding values are displayed on the right border of the figure)

Table 3 Mean scores of PEDQoL proxy scales in ATRT patients treated with proton therapy

\begin{tabular}{|c|c|c|c|c|c|c|}
\hline & $\begin{array}{l}\text { Physical Mean } \\
\text { Score }[ \pm \text { SD }] \\
\text { (number of } \\
\text { children) }\end{array}$ & $\begin{array}{l}\text { Emotion Mean } \\
\text { Score }[ \pm \mathrm{SD}] \\
\text { (number of } \\
\text { children) }\end{array}$ & $\begin{array}{l}\text { Social Mean } \\
\text { Score }[ \pm \text { SD] } \\
\text { (number of } \\
\text { children) }\end{array}$ & $\begin{array}{l}\text { Kindergarden/School } \\
\text { Mean Score }[ \pm \mathrm{SD}] \\
\text { (number of children) }\end{array}$ & $\begin{array}{l}\text { Psycho-social } \\
\text { Mean Score } \\
{[ \pm \mathrm{SD}] \text { (number of }} \\
\text { children) }\end{array}$ & $\begin{array}{l}\text { Total Mean } \\
\text { Score }[ \pm \text { SD }] \\
\text { (number of } \\
\text { children) }\end{array}$ \\
\hline $\begin{array}{l}\text { Baseline evaluation }^{\mathrm{a}} \\
(\mathrm{E} 1)^{\mathrm{c}}\end{array}$ & $\begin{array}{l}39.59[ \pm 22.31] \\
\quad(8)\end{array}$ & $\begin{array}{l}41.53[ \pm 18.98] \\
\quad(9)\end{array}$ & $\begin{array}{l}47.07[ \pm 28.44] \\
\quad(7)\end{array}$ & $56.25[ \pm 4.17]$ & $45.35[ \pm 16.91](7)$ & $\begin{array}{l}\mathbf{4 4 . 2 0}[ \pm 18.53] \\
\quad(8)\end{array}$ \\
\hline $\begin{array}{l}\text { Second evaluation }{ }^{\mathrm{b}} \\
\text { (E2) }^{\mathrm{d}}\end{array}$ & $\begin{array}{l}43.59[ \pm 21.03] \\
\quad(8)\end{array}$ & $\begin{array}{l}44.19[ \pm 21.04] \\
\quad(8)\end{array}$ & $\begin{array}{l}35.86[ \pm 26.79] \\
\quad(7)\end{array}$ & $62.50[ \pm 8.33]$ & $43.71[ \pm 15.43](7)$ & $\begin{array}{l}\text { 42.01 }[ \pm 17.84] \\
\text { (7) }\end{array}$ \\
\hline
\end{tabular}

Bold values are the mean of all scores for the various sub-scores

$S D$ Standard deviation

${ }^{\text {a }}$ Baseline, prior to PT

b approximately, 2 months after the completion of all therapy

c Number of E1 evaluation do not add to $n=15$, as not all proxy filled all domains

d Number of E2 evaluation do not add to $n=9$, as not all proxy filled all domains

death or end of follow-up for survival and tumor control endpoints. $\chi^{2}$ test, or the Fischer exact test when appropriate, was used to assess differences in patient distribution between groups. To assess variables influencing tumor control and OS and PFS, univariate analyses (using the logrank statistics at the $0.05 \propto$ level) were performed to evaluate clinical (gender, age, tumor location, tumor size) and therapeutic (type of surgery, concomitant chemotherapy) factors. All $p$ values were based on a 2-sided hypothesis. The statistical analyses were performed on the SPSS statistics program, version 22 (IBM Corporation, Armonk, New York, USA).

\section{Results}

Of those patients with residual disease before PT ( $n=7), 2$ $(28.2 \%)$ patients achieved complete response,
11.4-13.8 months after PBS. Three (43.0 \%) patients had stable disease (mean diameter decrease, $7.0 \%$; range 0.9-13.0) after irradiation and two other $(28.5 \%)$ patients presented with PD, exhibiting a significant increase in tumor diameter (increase of +48.9 and $+57.1 \%$, respectively). No pseudoprogression was observed.

After a median follow-up of 33.4 months (range 9.7-69.2) for all patients and 37.1 months (range 17.8-69.2) for living patients, 6 patients have experienced tumor recurrence or progression. The estimated 2-year PFS was $66.0 \%$ (CI $95 \%$ 41.7-90.3). Three patients with supratentorial tumors $(p=0.04)$ presented with a local failure (LF), 8.7-18.4 (median, 10.7) months after PT (Table 2). The estimated 2-year LF-free survival was $78.0 \%$ (CI $95 \%$ 55.7-100). Four other patients presented with distant brain failure (DBF), 1.3-28.5 (median 7.1) months after PT. The estimated 2-year DBF-free survival was $76.6 \%$ (CI $95 \%$ 43.9-100). These failures were 


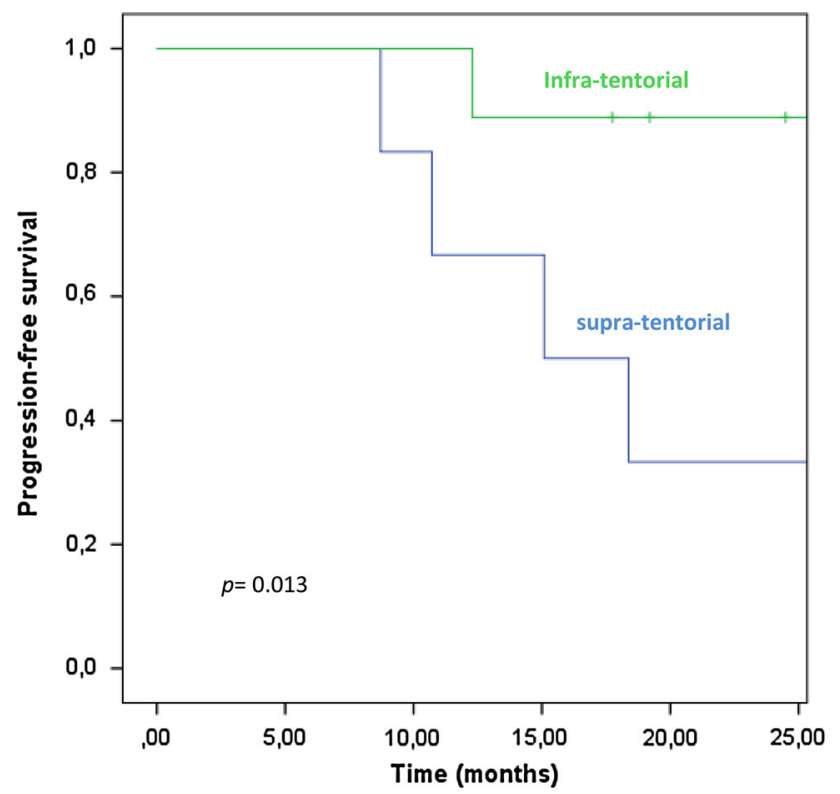

Fig. 2 Progression-free survival in 15 patients with supra- $(n=6)$ and infratentorial $(n=9)$ ATRT treated with proton therapy

observed in the ponto-cerebellar angle in 2 patients and another two patients presented with brain leptomeningeal recurrences. All four DBFs occurred in patients with ATRTs in supratentorial location $(p=0.01)$. Spinal failures were observed in 2 patients (infratentorial: $n=1$; supratentorial: $n=1$ ), 12.3 and 37.1 months after PT (Table 2). The estimated 2-year spinal PFS was $92.9 \%$ (CI $95 \%$ 79.4-100). Combined treatment failures (LF and DBF, $n=2$; DBF and SF, $n=1$ ) were observed in 3 patients.

Salvage 'curative' therapy was administered to $2(33 \%)$ patients and best supportive care was proposed to another 4 (67\%) patients (Table 2). For the former patients, surgery and chemotherapy was administered to one patient and surgery, PT (second irradiation) and chemotherapy was administered to the other patient. These two patients survived 23.5 and 59.3 months, respectively.

Six deaths were caused by LF, DBF or SF (Table 2). The estimated 2-year OS was $64.6 \%$ (CI $95 \%$ 39.3-89.9).

On univariate analysis, tumor location was statistically significant for both OS and PFS (Fig. 2). The 2-year OS and PFS for infra- and supratentorial ATRTs were both 88.9 and $33.3 \%$, respectively (OS: $p=0.012$; PFS: $p=0.013)$. The latter localization was observed usually in younger patients. Sixty seven percent and $33 \%$ of patients $<12$ months presented with a supra- and infratentorial ATRT, respectively $(p=0.53)$. There was a statistical trend toward significance for the type of surgical resection. The 2-year OS for patients with a complete resection and those with a subtotal resection/biopsy was 85.7 and $46.9 \%$, respectively $(p=0.067)$. For PFS, the 2-year survival rate for patients with a complete resection and those with a subtotal resection/biopsy was 85.7 and $50.0 \%$, respectively $(p=0.084)$. Gender (OS: $p=0.42$; PFS: $p=0.45$ ), tumor size ( $<5$ vs. $\geq 5 \mathrm{~cm}$; OS: $p=0.49$; PFS: $p=0.37)$, the administration of concomitant chemotherapy (yes vs. no; OS: $p=0.35$; PFS: $p=0.43$ ) and age of the patient ( $<12$ vs. $\geq 12$ months; OS $p=0.79$; PFS: $p=0.70$ ) was not significant for both OS or PFS.

PT was well tolerated. PT was delivered without any interruption for all patients. Only $2(13 \%)$ patients had a decreased performance status of WHO 2 after PT. Bone marrow grade 1 and 2 acute toxicity was observed in 11 and 2 children, respectively. Alopecia was observed in all children. All but one child had grade 1-2 erythema. No grade $\geq 3$ acute toxicity was observed. Two $(13 \%)$ children presented with late toxicity (grade 1 and 4 motor dysfunction, respectively). In one of these patients, radionecrosis was observed. This young girl of 22.8 months of age at the time of diagnosis presented with tetra-paresis and a radio-necrosis in the mesial aspect of the temporal lobe and brainstem. She is currently alive 43.5 months after PBS. In this series, the estimated 2-year TFS was $90 \%$ (CI $95 \%$ 71.4-100).

All parents filled the PedsQoL proxy evaluation at baseline (E1). A second evaluation (E2) was obtained at 2 months of follow-up for $9(60 \%)$ children in at least one QoL-metric. The results are detailed in Table 3. Only minimal changes in Physical and Emotion function, as well as in the Summary scores, were observed. Noteworthy, slightly higher negative variations in Social function were observed. Using PedsQoL proxy, these results suggest that PT did not negatively impact the overall QoL of these ATRT patients treated with PT.

\section{Discussion}

We report outcomes in a cohort of 15 children treated with PT for ATRT and to the best of our knowledge, the present series is the only series ever published on this tumor entity using PT and PBS. Protons have a depth-dose distribution that is characterized by a narrow Bragg peak and a sharp distal fall-off beyond this range. PBS, as opposed to passive scattering, enables to increase the dose conformity of proton radiation, proximal to the target volume. As such, the brain-integral dose and the radiation administered to CNS critical structures in vicinity of the target may be decreased with PBS, which could lead in a decrease of radiation-induced toxicity. A previous series reported on the outcome of 10 ATRT patients treated with passive scattering PT [9]. As such, PT have been successfully administered to children with brain tumors [10, 11], for whom the young age during treatment and the proximity of critical structures make protons an exciting radiation 
modality. Due to the small number of proton facilities worldwide, the poor prognosis associated with this tumor entity and the higher costs associated with protons, PT is usually not administered to these young children. Given the increased number of constructed proton facilities and the improved overall prognosis of these patients, it is of paramount importance that this treatment modality should be indeed considered for these patients. With an estimated 2 year-survival of $66.0 \%$ of patients in our series, a substantial number of ATRT patients could benefit of this highly conformal treatment with no serious toxicity or decreased in QoL. As for the treatment costs, a number of cost-effectiveness analyses have suggested that PT for brain tumors in children were associated with lower costs and higher quality-adjusted life years [12, 13]. Some series, using a Markov model, have even shown that protons could be cost-saving for medulloblastoma [14].

The higher frequency of ATRT in infants complicates substantially therapy due to the customary avoidance of RT in some groups in this age category [15]. Radiation therapy remains however one of the most important treatment modality for this challenging tumor occurring in very young children. In the Korean study, tumor relapse or progression during induction chemotherapy was seen in the majority $(56 \%)$ of patients treated with a HD chemotherapy regimen [16]. The same percentage (55\%) of tumor progression or relapse during chemotherapy was observed in the St Jude series [17]. Two studies have shown undisputedly the importance of RT. A retrospective review of 31 patients followed up at St. Jude Children's Research Hospital suggested that children who received chemotherapy and radiotherapy had a better outcome [18]. Likewise, the results of the baby Pediatric Oncology Group 2 study, addressing the efficacy of HD chemotherapy to avoid or delay radiation in young children, showed that all 36 ATRT patients included in this protocol ultimately died of their disease. The median unreported OS was only 6.7 months [19].

The timing of RT has been even less studied in the available literature. Two important series have however been published in Taiwan and in the US. Chen et al. reported retrospectively on 17 patients treated with chemotherapy and mainly CSI [20]. The median OS was 17 months and multivariate analysis revealed a significant relationship between overall survival and the time interval between surgery and radiotherapy initiation $(p=0.031)$. Moreover, the time to radiation completion $(p=0.047)$ was also significantly associated with OS. Likewise, the St. Jude group reported on 31 patients (median age 2.3 years) treated with focal RT or with the addition of CSI [17]. Using a Cox regression model, the authors have shown that children receiving delayed ( $>1$ month postoperatively) RT were more likely to experience local failure (hazard ratio
1.23, $p=0.007$ ) than those who received immediate postoperative RT. As such, it is of critical importance to have RT timely delivered after the initial surgery.

The pathogenesis of ATRT is still poorly understood and molecular markers for risk-adapted patient stratification are not available. As such, we rely on a number of crude clinical prognosticators. The prognostic impact of ATRT localization is debated. This clinical prognostic factor has not been analyzed in numerous series [16, 17, 20, 21]. In a tumor registry US series, $8(58 \%)$ of the long term survivors had supratentorial tumors, but no formal analysis was made for this parameter [1]. In another population-based tumor registry Austrian study, tumor localization was not associated with OS $(p=0.49)$ [3]. The same finding was observed in a recently published large retrospective Canadian series [22] and in a meta-analysis of observational studies performed by Athale et al. [4].. In the HIT series however, overall survival was significantly higher $(p=0.003)$ in patients with supratentorial tumor localization ( 3 year OS: $38 \pm 10 \%$ ) compared to patients with infratentorial tumors (3 year OS: $5 \pm 4 \%$ ) [23]. Conversely, the calculated median OS for patients with supratentorial ATRTs was 24 months and significantly $(p=0.04)$ shorter than the survivorship of patients with posterior fossa tumors (median OS not yet reached) in the Dana-Farber study [15]. In our study, we also observed that the survival rate of patients with posterior-fossa tumors was better by a factor of approximately three when compared to those with supratentorial tumors $(p=0.012)$. Possible explanations for this finding include imbalances between the two groups with respect to known and unknown baseline prognostic factors, imbalances in the use of second therapies if any, statistical chance or a real tumour localization difference.

Younger age is usually an adverse prognostic factor for ATRT patients. In the HIT series, children younger than the median age at diagnosis (15 months) had a lower eventfree survival $(p=0.044)$ and OS $(p=0.002)$ than older children [23]. Likewise, in a retrospective analysis of 37 patients treated at St Jude, the estimated 2-year OS was $17 \pm 8$ and $89 \pm 11 \%(p=0.009)$ for children younger and older than 36 months, respectively. The corresponding value for the event-free survival was $11 \pm 6$ and $78 \pm 14 \%$ and this difference was highly significant ( $p=0.0001$ ) [18]. A poorer outcome was also observed in children $<12$ months of age in the Canadian retrospective analysis but this trend did not reach statistical significance $(p=0.06)$ [22]. Age was however not a significant factor $(p=0.4)$ in the Dana-Farber series [15], nor was it a prognosticator in the aforementioned tumor registry Austrian study [3] and meta-analysis series [4]. In our series, we did not find that age was a significant prognostic factor. Younger ATRT patients in our series had usually a 
supratentorial tumor and older patients an infratentorial ATRT.

We have observed that the OS for the 7 patients who had complete resection was 31.7 months (range 12.4-69.2), compared to the OS of 21.4 months (range 9.7-59.3) for the subset with a partial resection/biopsy $(p=0.067)$. The same statistical trend for event-free survival was observed in the HIT series [23]. Likewise, the extent of the initial tumor resection was correlated with OS in the early and recently published St Jude series but not significantly so $[17,18]$. This is in line with the Dana-Farber series which reported a 2-year OS of $91 \pm 9 \%$ for patients who achieved a gross total resection, whereas the reported median OS of less than gross totally resected patient was 18 months ( $p=0.004$ ) [15]. In the Austrian tumor registry study, extent of resection was also significantly associated with improved survival ( $p=0.013$ ) [3]. As a result of the published data, we would strongly recommend that an aggressive surgical strategy be undertaken, including second-look surgery, to best achieve gross total resection.

This is also the first analysis to report the QoL outcome of children with ATRTs after PT. Pediatric malignant brain tumors are often associated with physical, cognitive, psychological and behavioral difficulties that may affect substantially the QoL of children and their families. In this study, we analyzed the parental report of QoL in children with ATRT. Parent-proxy reported QoL for children with various chronic CNS disease, not limited to but including cerebral palsy [24], obsessive-compulsive [25] and attention-deficit hyperactivity disorders [26] have been successful in detecting children's impairment with these conditions. For children with cancer, this proxy-reporting has not been fully assessed and validated. As such, great care should be taken not to over-interpret these data in this small cohort of very young children with a rare brain tumor. Notwithstanding the lack of definite proof of validity in parental report for this challenging tumor, our data suggests that PT may not have a detrimental effect on the QoL of these patients. The increased mean scores of $3 / 5$ domains after therapy may suggest an improvement of QoL, when compared to baseline scores (Table 3).

There were several limitations of our study. First, the study design was retrospective in nature and thus lacked complete data for certain variables such as cumulative chemotherapy dose. Second, the small sample size of 15 patients limited the statistical power to detect associations between tumour progression and some of the clinical factors examined. A third limitation is the possible underreporting of treatment-related toxicity. Although every patient was jointly followed up at 2-3-monthly intervals by both their treating medical and radiation oncologist, it is still possible, albeit unlikely, that asymptomatic patients may have had radiographic evidence of radiation-induced toxicity that went unreported, especially in a retrospective analysis. Finally, no central review of pathology was performed for the analysis, but the loss of INI-1 expression for all patients (Table 1) in tumours that had all histological characteristics of ATRT mitigates somewhat this disclaimer.

\section{Conclusions}

Our data suggests that PBS PT is an effective treatment for young children with ATRT. After PT, with or without concomitant chemotherapy, two third of the patients survived $>2$ years. The acute toxicity was limited and our prospective parental-proxy reporting data do not suggest a decrease of QoL of these very young patients. Late toxicity was unusual. Supratentorial tumor localization, occurring usually in very young patients, was a negative prognostic factor in our series.

Acknowledgments We thank Carmen Teske (University of Münster), Anna Wiener Wellauer (PSI), April Siegwolf (PSI) and Beate Schulz (PSI) for providing data management.

Conflict of interest The author \& co-authors have no potential conflict of interest.

\section{References}

1. Hilden JM, Meerbaum S, Burger P, Finlay J, Janss A, Scheithauer BW, Walter AW, Rorke LB, Biegel JA (2004) Central nervous system atypical teratoid/rhabdoid tumor: results of therapy in children enrolled in a registry. J Clin Oncol 22:2877-2884. doi:10.1200/JCO.2004.07.073

2. Biegel JA (2006) Molecular genetics of atypical teratoid/rhabdoid tumor. Neurosurg Focus 20:E11

3. Woehrer A, Slave I, Waldhoer T, Heinzl H, Zielonke N, Czech T, Benesch M, Hainfellner JA, Haberler C, Austrian Brain Tumor R (2010) Incidence of atypical teratoid/rhabdoid tumors in children: a population-based study by the Austrian Brain Tumor Registry, 1996-2006. Cancer 116:5725-5732. doi:10.1002/cncr.25540

4. Athale UH, Duckworth J, Odame I, Barr R (2009) Childhood atypical teratoid rhabdoid tumor of the central nervous system: a meta-analysis of observational studies. J Pediatr Hematol Oncol 31:651-663. doi:10.1097/MPH.0b013e3181b258a9

5. Brodin NP, Munck Af Rosenschold P, Aznar MC, Kiil-Berthelsen A, Vogelius IR, Nilsson P, Lannering B, Bjork-Eriksson T (2011) Radiobiological risk estimates of adverse events and secondary cancer for proton and photon radiation therapy of pediatric medulloblastoma. Acta Oncol 50:806-816. doi:10.3109/ 0284186X.2011.582514

6. Weber DC, Rutz HP, Pedroni ES, Bolsi A, Timmermann B, Verwey J, Lomax AJ, Goitein G (2005) Results of spot-scanning proton radiation therapy for chordoma and chondrosarcoma of the skull base: the Paul Scherrer Institut experience. Int J Radiat Oncol Biol Phys 63:401-409

7. Lomax AJ, Bohringer T, Bolsi A, Coray D, Emert F, Goitein G, Jermann M, Lin S, Pedroni E, Rutz H, Stadelmann O, 
Timmermann B, Verwey J, Weber DC (2004) Treatment planning and verification of proton therapy using spot scanning: initial experiences. Med Phys 31:3150-3157

8. Varni JW, Burwinkle TM, Katz ER, Meeske K, Dickinson P (2002) The PedsQL in pediatric cancer: reliability and validity of the pediatric quality of life inventory generic core scales, multidimensional fatigue scale, and cancer module. Cancer 94:20902106

9. De Amorim Bernstein K, Sethi R, Trofimov A, Zeng C, Fullerton B, Yeap BY, Ebb D, Tarbell NJ, Yock TI, MacDonald SM (2013) Early clinical outcomes using proton radiation for children with central nervous system atypical teratoid rhabdoid tumors. Int $\mathbf{J}$ Radiat Oncol Biol Phys 86:114-120. doi:10.1016/j.ijrobp.2012. 12.004

10. Jimenez RB, Sethi R, Depauw N, Pulsifer MB, Adams J, McBride SM, Ebb D, Fullerton BC, Tarbell NJ, Yock TI, Macdonald SM (2013) Proton radiation therapy for pediatric medulloblastoma and supratentorial primitive neuroectodermal tumors: outcomes for very young children treated with upfront chemotherapy. Int J Radiat Oncol Biol Phys 87:120-126. doi:10.1016/j. ijrobp.2013.05.017

11. MacDonald SM, Yock TI (2010) Proton beam therapy following resection for childhood ependymoma. Child's nerv syst 26:285-291. doi:10.1007/s00381-009-1059-4

12. Mailhot Vega RB, Kim J, Bussiere M, Hattangadi J, Hollander A, Michalski J, Tarbell NJ, Yock T, MacDonald SM (2013) Cost effectiveness of proton therapy compared with photon therapy in the management of pediatric medulloblastoma. Cancer 119:4299-4307. doi:10.1002/cncr.28322

13. Hirano E, Fuji H, Onoe T, Kumar V, Shirato H, Kawabuchi K (2014) Cost-effectiveness analysis of cochlear dose reduction by proton beam therapy for medulloblastoma in childhood. J Radiat Res 55:320-327. doi:10.1093/jrr/rrt112

14. Lundkvist J, Ekman M, Ericsson SR, Jonsson B, Glimelius B (2005) Cost-effectiveness of proton radiation in the treatment of childhood medulloblastoma. Cancer 103:793-801. doi:10.1002/ cncr. 20844

15. Chi SN, Zimmerman MA, Yao X, Cohen KJ, Burger P, Biegel JA, Rorke-Adams LB, Fisher MJ, Janss A, Mazewski C, Goldman S, Manley PE, Bowers DC, Bendel A, Rubin J, Turner CD, Marcus KJ, Goumnerova L, Ullrich NJ, Kieran MW (2009) Intensive multimodality treatment for children with newly diagnosed CNS atypical teratoid rhabdoid tumor. J Clin Oncol 27:385-389. doi:10.1200/JCO.2008.18.7724

16. Park ES, Sung KW, Baek HJ, Park KD, Park HJ, Won SC, Lim do H, Kim HS (2012) Tandem high-dose chemotherapy and autologous stem cell transplantation in young children with atypical teratoid/rhabdoid tumor of the central nervous system. J Korean Med Sci 27:135-140. doi:10.3346/jkms.2012.27.2.135

17. Pai Panandiker AS, Merchant TE, Beltran C, Wu S, Sharma S, Boop FA, Jenkins JJ, Helton KJ, Wright KD, Broniscer A, Kun
LE, Gajjar A (2012) Sequencing of local therapy affects the pattern of treatment failure and survival in children with atypical teratoid rhabdoid tumors of the central nervous system. Int J Radiat Oncol Biol Phys 82:1756-1763. doi:10.1016/j.jirobp. 2011.02.059

18. Tekautz TM, Fuller CE, Blaney S, Fouladi M, Broniscer A, Merchant TE, Krasin M, Dalton J, Hale G, Kun LE, Wallace D, Gilbertson RJ, Gajjar A (2005) Atypical teratoid/rhabdoid tumors (ATRT): improved survival in children 3 years of age and older with radiation therapy and high-dose alkylator-based chemotherapy. J Clin Oncol 23:1491-1499. doi:10.1200/JCO.2005.05. 187

19. Ginn KF, Gajjar A (2012) Atypical teratoid rhabdoid tumor: current therapy and future directions. Frontiers oncol 2:114. doi:10.3389/fonc.2012.00114

20. Chen YW, Wong TT, Ho DM, Huang PI, Chang KP, Shiau CY, Yen SH (2006) Impact of radiotherapy for pediatric CNS atypical teratoid/rhabdoid tumor (single institute experience). Int J Radiat Oncol Biol Phys 64:1038-1043. doi:10.1016/j.ijrobp.2005.10.001

21. Chen ML, McComb JG, Krieger MD (2005) Atypical teratoid/ rhabdoid tumors of the central nervous system: management and outcomes. Neurosurg Focus 18:E8

22. Lafay-Cousin L, Hawkins C, Carret AS, Johnston D, Zelcer S, Wilson B, Jabado N, Scheinemann K, Eisenstat D, Fryer C, Fleming A, Mpofu C, Larouche V, Strother D, Bouffet E, Huang A (2012) Central nervous system atypical teratoid rhabdoid tumours: the Canadian paediatric brain tumour consortium experience. Eur J Cancer 48:353-359. doi:10.1016/j.ejca.2011. 09.005

23. von Hoff K, Hinkes B, Dannenmann-Stern E, von Bueren AO, Warmuth-Metz M, Soerensen N, Emser A, Zwiener I, Schlegel PG, Kuehl J, Fruhwald MC, Kortmann RD, Pietsch T, Rutkowski $S$ (2011) Frequency, risk-factors and survival of children with atypical teratoid rhabdoid tumors (AT/RT) of the CNS diagnosed between 1988 and 2004, and registered to the German HIT database. Pediatr Blood Cancer 57:978-985. doi:10.1002/pbc. 23236

24. Davis E, Mackinnon A, Waters E (2012) Parent proxy-reported quality of life for children with cerebral palsy: is it related to parental psychosocial distress? Child Care Health Dev 38:553-560. doi:10.1111/j.1365-2214.2011.01267.x

25. Lack CW, Storch EA, Keeley ML, Geffken GR, Ricketts ED, Murphy TK, Goodman WK (2009) Quality of life in children and adolescents with obsessive-compulsive disorder: base rates, parent-child agreement, and clinical correlates. Soc Psychiatry Psychiatr Epidemiol 44:935-942. doi:10.1007/s00127-009-00139

26. Matza LS, Secnik K, Rentz AM, Mannix S, Sallee FR, Gilbert D, Revicki DA (2005) Assessment of health state utilities for attention-deficit/hyperactivity disorder in children using parent proxy report. Qual Life Res 14:735-747 